\title{
Selecting asteroids for a targeted spectroscopic survey ${ }^{\star}$
}

\author{
D. A. Oszkiewicz, ${ }^{1}$, T. Kwiatkowski ${ }^{1}$, T. Tomov ${ }^{2}$, M. Birlan ${ }^{3,4}$, S. Geier ${ }^{5}$, A. Penttilä ${ }^{6}$, and M. Polińska ${ }^{1}$ \\ 1 Astronomical Observatory Institute, Faculty of Physics, A. Mickiewicz University, Słoneczna 36, 60-286 Poznań, Poland \\ e-mail: dagmara.oszkiewicz@gmail.com \\ 2 Nicholaus Copernicus University, ul. Gagarina 11, 87-100 Toruń, Poland \\ 3 IMCCE - Paris Observatory - UMR 8028 CNRS 77, av. Denfert-Rochereau, 75014 Paris, France \\ 4 Astronomical Institute of the Romanian Academy, Strada Cutitul de Argint 5, 040557 Bucureti, Romania \\ 5 Nordic Optical Telescope, Apartado 474, 38700 Santa Cruz de La Palma, Spain \\ ${ }^{6}$ Division of Geophysics and Astronomy, Department of Physics, PO Box 64, 00014 University of Helsinki, Finland
}

Received 13 December 2013 / Accepted 15 August 2014

\begin{abstract}
Context. Asteroid spectroscopy reflects surface mineralogy. There are a few thousand asteroids whose surfaces have been observed spectrally. Determining their surface properties is important for many practical and scientific applications, such as developing impact deflection strategies or studying the history and evolution of the solar system and planet formation.

Aims. The aim of this study is to develop a preselection method that can be used to search for asteroids of any taxonomic complex. The method could then be utilized in multiple applications, such as searching for the missing V-types or looking for primitive asteroids. Methods. We used the Bayes Naive Classifier combined with observations obtained in the course of the Sloan Digital Sky Survey and the Wide-field Infrared Survey Explorer surveys, as well as a database of asteroid phase curves for asteroids with a known taxonomic type. With this new classification method, we selected a number of possible V-type candidates. Some of the candidates were then spectrally observed at the Nordic Optical Telescope and South African Large Telescope.

Results. We developed and tested the new preselection method. We found three asteroids in the mid-to-outer main belt that probably have differentiated types. Near-infrared observations are still required to confirm this discovery. As in other studies we found that V-type candidates cluster around the Vesta family and are rare in the mid-to-outer main belt.

Conclusions. The new method shows that even largely explored large databases when combined could still be exploited further in, for example, solving the missing dunite problem.
\end{abstract}

Key words. methods: statistical - techniques: spectroscopic - minor planets, asteroids: general

\section{Introduction}

Laboratory analysis of the different groups of meteorites has suggested that there must have once been at least 50 to 100 large (150-300 km in diameter) differentiated parent bodies (into distinct mineralogical layers, that is core, mantle, and crust). Those bodies went though a number of disruptive collisions shedding material from their different mineralogy layers and creating hypothetical differentiated asteroid families. Members of those families should have similar orbits and various mineralogical composition across the family. In particular, they should contain asteroidal fragments originating in iron core, silicate/basaltic mantle, and basaltic crust regions of their parent bodies.

However, to date solar system evolution theory, meteoritic evidence, and current observations of asteroids are unmatchable. On one hand, laboratory examination of meteorites indicates that there were multiple parent bodies. In particular, the diverse thermal history (Goldstein \& Short 1967; Kelley \& Gaffey 2009) of iron meteorites indicates at least 50 parent bodies cooling at different rates. Analysis of oxygen isotopic ratios (Scott et al. 2009) in the meteorites from the howardites-eucrites-diogenites (HED) group (commonly believed to be related to the asteroid (4) Vesta) also shows evidence of at least five other than Vesta differentiated parent bodies. However, to date the only presently

\footnotetext{
* Tables 6 and A.1 are only available at the CDS via anonymous ftp to cdsarc.u-strasbg. fr $(130.79 .128 .5)$ or via http://cdsarc.u-strasbg.fr/viz-bin/qcat?J/A+A/572/A29
}

existing asteroid family that bears evidence of a differentiated parent body is the Vesta family. Present observations of other known asteroid families do not support the hypothesis of multiple parent bodies. Current spectroscopic observations show a deficiency of basaltic (originating in the mantle and crust) asteroids as compared to the estimations based on meteorite samples (for example, Bottke et al. 2006 or Burbine et al. 1996).

Furthermore, most of the asteroid families seem to be internally homogeneous. The reflectance spectroscopy of family members shows the homogeneity (Mothé-Diniz et al. 2005) of their surfaces, indicating similar mineralogy within the family. Members of the same asteroid family also often have similar physical properties, such as color indices (Jurić et al. 2002), albedos, and photometric parameters (Oszkiewicz et al. 2011). This kind of surface uniformity suggests similar mineralogical composition and the homogeneity of the parent body. Asteroid families created during collisions of differentiated parent bodies should contain asteroidal fragments of varying composition (Lazzaro 2009).

In addition, as mentioned before the only asteroid family that is presently confirmed to originate in a differentiated parent body is the Vesta family. The Vesta family arose from a collisional event that also formed a large crater on the surface of asteroid (4) Vesta. Recently NASA's Dawn spacecraft visited Vesta and confirmed the existence of iron core by gravitational field measurements (Russell et al. 2012). Though the presence of iron core was confirmed, the details of Vesta's differentiation are elusive. 
Two types of Vesta differentiation models are commonly discussed. The magma-ocean models generate eucritic crust overlying diogenite layer, with olivine-rich mantle and a metallic core (for example Righter et al. 1997). An equivalent model (for example Barrat et al. 2010) posits that Vesta formed with pockets of magma slowly crystallizing beneath the surface, leading to diogenitic plutons at the crust-mantle boundary or within the basaltic crust. Both of those models have recently been challenged by the findings provided by the Dawn mission. The main challenges occur because the observed amounts and distribution of the olivine material on Vesta is inconsistent with the models (Ammannito et al. 2013).

In general, partial differentiation models are also considered possible for some bodies. For example the high bulk density of Lutetia exceeds the density of most common chondric meteorite groups (Weiss et al. 2013), suggesting that partial differentiation may have led to the creation of metallic core covered by a primitive chondritic crust. This finding is also supported by earlier studies (Carporzen et al. 2011) that show an existing remnant magnetic field in carbonaceous chondrites due to core dynamo. Those findings may suggest that other asteroids with chondritic surfaces may in fact be differentiated and have a metallic core (Weiss et al. 2013).

The exact reasons for the discrepancies between the meteoritic evidence and the observational data of asteroids in the main belt still need to be explained. Numerous hypotheses are being tested with the most prevailing being that the differentiated parent bodies were "battered to bits" during collisions and are below our current spectroscopic reach Burbine et al. (1996). Another possible explanation is that space-weathering processes (Chapman 2004) could have erased the indicative spectroscopic features of V-type asteroids and made them look like S-type objects. Shock waves propagated during collisions could also hide characteristic spectral features of some materials. For example, laboratory spectroscopy of Chelyabinsk meteorites shows that shocked fragments of ordinary chondrites (known to originate in S-type asteroids) can have a spectrum similar to a C-type object (Kohout et al. 2013).

That strong shocking of material mask the absorption bands of HED meteorites and their analog V-type asteroids has yet to be tested. Another possibility is that some of the material was somehow selectively removed from the main belt - a hypothesis needs to be investigated. Evidence of such processes can be seen in other planetary systems. In particular, the so-called "polluted" spectra of white dwarfs have been found to indicate differentiated objects accreting onto the star (Jura et al. 2012; Dufour et al. 2010). It has also been suggested that after collisions, fragments of the differentiated parent bodies reaccumulated, covering up the basaltic material. Last but not least, our traditional understanding of the differentiation process could be incorrect, perhaps pointing toward the idea of partial differentiation as a more common process in the early solar system (Weiss et al. 2013). No matter what the real explanation is, the search for more evidence of differentiation in the main belt is ongoing.

Several surveys and asteroid selection methods that target the possible remnants of the differentiated material in the asteroid belt have been published. Most of the selection methods focus on finding V-type candidate asteroids using the photometric colors obtained by the Sloan Digital Sky Survey (SDSS) (Ivezic et al. 2002). For example, Roig \& Gil-Hutton (2006) used the SDSS color photometry in combination with principal components analysis (PCA) to select candidates for a spectral survey of $\mathrm{V}$-type asteroids. The basic idea was to identify the phase-space area where the known V-type asteroids reside and then classify all other asteroids that reside in this area as possible V-type candidates. Moskovitz et al. (2008a) presents another method. The method relies on selecting objects having similar photometric colors to those of Vesta family asteroids. Most of the Vesta family asteroids are of V-type, therefore selecting asteroids having similar colors should result in finding other V-type candidates. Carvano et al. (2010) derived a new classification scheme for SDSS asteroid colors and investigated the distributions of the new classes in the main belt. In particular, the Vp class showed a clustering around the Vesta family and also a scatter in the mid and outer main belt. Another method by Solontoi et al. (2012) primarily uses the $i-z$ color index to find candidate V-type asteroids. In that method all the asteroids fulfilling the criteria $i-z<-0.2$ were clasified as possible V-type candidates.

In this study we propose a new selection method that can suggest candidate asteroids for each taxonomic complex. The proposed method assigns spectral complex probability to each asteroid under examination. In contrast to other methods, we use multiple databases containing different indicators (here and after features) of spectral complex. In particular, we use the Sloan Digital Sky Surveys (SDSS) photometric database, phase function parameter $G_{12}$ database, and Wide-field Infrared Survey Explorer (WISE) albedo database. Several asteroids selected by the new method are then targeted using SALT and NOT telescopes. Results of those observations are reported. The selection method is compared to similar earlier studies. As opposed to the other methods, the technique proposed here can predict objects of any taxonomic complex. This can be especially useful in applications other than searching for differentiated bodies and their remnants. For example, various missions to near earth asteroids look for primitive C-type objects as possible targets.

In Sect. 2 we present this novel selection method and possible V-type candidates. In Sect. 3 we discuss the observations and data reduction. In Sect. 4 we present our results. Section 5 contains conclusions and discussion.

\section{Selection method}

To compute the probability of an object having a particular taxonomic complex, we use all the information on asteroid physical properties available to date in large databases. Previous selection methods focused on using solely the photometric measurements from the Moving Object Catalog (MOC) obtained in the course of the Sloan Sky Digital Survey (SDSS). In our method we extend the inference by also using other databases: the database of asteroid phase curves (Oszkiewicz et al. 2011) and the asteroid albedos database from the Wide-Field Infrared Survey Explorer (WISE) (Masiero et al. 2011). In our method, all those features combined (albedos, slope parameters, and SDSS reflectance fluxes) determine the probability of an asteroid having a particular class or complex.

Adding additional features is beneficial for the purposes of preliminary classification in several ways. First, the initial classification can be made for objects that could not be classified based solely on SDSS. This includes objects not observed in the course of the SDDS, but observed by the WISE mission with the $G_{12}$ parameter computed. Second, certain features segregate particular classes better than others. For example the $\mathrm{C}$ and $\mathrm{X}$ types cannot be totally segregated, given the SDSS data only because the typical differences between the typical SDSS albedos of both classes are within the $10 \%$ error of the data (Roig \& Gil-Hutton 2006). On the other hand, the $G_{12}$ parameter cannot distinguish between $\mathrm{S}$ and $\mathrm{X}$ types due to large distribution overlap, but $\mathrm{C}$ types are 
better segregated from both $\mathrm{S}$ and $\mathrm{X}$ types (Oszkiewicz et al. 2012). Combing the SDSS data and $G_{12}$ could therefore lead to a more reliable segregation of the $\mathrm{C}$ types.

SDSS data points are available for about 100000 known objects, albedos for around 100000 objects, and slope parameters for about 500000 objects. Not all of the data are mutually inclusive, so the probability has to be sometimes computated based on a single feature. This is ofcourse less reliable than when all the features are available. Some of the data points contain large errors, which also influences the reliability of the resulting probability computation. In contrast to other methods, we decided not to remove the data points containing large errors in order not to discriminate against possible basaltic candidates. This of course has an impact on the method efficiency, but we consider it a trade-off worth making. The errors can be included in the probability computation. In the subsequent sections, we explain the classification algorithm, the training dataset, and uncertainties. To compute the probability that an object belongs to a specific taxonomic complex, we first estimate its probability of belonging to each taxonomic class. The complex probability is then computed by integrating over all the classes in a given complex.

\subsection{Classification algorithm}

In our classification algorithm, we make use of one of the most common classifier in machine learning, the naive Bayes classifier. In this classifiers, the prediction of a class of an object is made based on the features of the objects that are considered independent of each other (Russell et al. 2002). This means that each of the object features or attributes contributes separately to identifying the class, therefore only the variances of the features have to be known, without computing the correlations between the features and the full covariance matrix. Even though the independence assumptions can sometimes be wrong (which they clearly are in the case of photometric data, albedo, and phase curve parameters), naive Bayes classifiers have already proven to be a very efficient tool in many practical applications. One noteworthy advantage of Bayes classifiers is that they can handle missing data by integrating over all possible values of the feature.

In general, the probability of each class can be computed as an a posteriori probability of the class given some specific values of object's features $\mathbf{F}=\left[F_{1}, \ldots, F_{n}\right]$ for $j=1, \ldots, n$ (Russell et al. 2002):

$P\left(C \mid F_{1}, \ldots, F_{n}\right)=\alpha P(C) \prod_{j=1}^{n} P\left(F_{j} \mid C\right)$,

where:

- $P\left(C \mid F_{1}, \ldots, F_{n}\right)$ is the a posteriori probability of a class, given the object's features or attributes $F_{1}, \ldots ., F_{n}$;

- $\alpha$ is the normalization constant;

- $P(C)$ is the a priori probability of a class that can be estimated as a frequency of specific class (the so-called informative prior) or assumed uniform among all the classes (the so-called weakly informative prior),

- $P\left(F_{j} \mid C\right)$ is the conditional probability of a feature $F_{j}$ assuming that the object is of a given class $C$.

This can be expanded to

$$
\begin{array}{r}
P\left(C \mid F_{1}, \ldots, F_{n}\right)=\alpha P(C) P\left(P_{V} \mid C\right) P\left(G_{12} \mid C\right) \\
P\left(F_{g} \mid C\right) P\left(F_{r} \mid C\right) P\left(F_{i} \mid C\right) P\left(F_{z} \mid C\right),
\end{array}
$$

where $P_{V}$ is WISE albedo, $G_{12}$ is phase curve parameter, and $F_{g}$, $F_{r}, F_{i}, F_{z}$ are the SDSS albedos (or relative reflectance values). The $F_{u}$ reflectance flux is omitted from classification - please see Sect. 2.2 for explanation. For example, the probability of an object being of a $\mathrm{V}$ class could be written as

$P(C=V \mid F)=\alpha P(C=V) P\left(p_{V} \mid C=V\right) P\left(g_{12} \mid C=V\right)$

$P\left(f_{g} \mid C=V\right) P\left(f_{r} \mid C=V\right) P\left(f_{i} \mid C=V\right) P\left(f_{z} \mid C=V\right)$,

where $p_{V} g_{12} f_{g} f_{r} f_{i} f_{z}$ are specific albedo, phase curve parameter, and relative flux values of an object, and the shapes of probability density functions $P$ are known from templates for a typical $V$ class object. Similarly, the probabilities for other taxonomic classes can be computed. The probability that an object is of a certain complex rather than of a specific class is computed by integrating over all possible classes in a given complex. For example, for the $S$ complex the probability could be computed as

$P\left(C=S-\right.$ complex $\left.\mid F_{1}, \ldots, F_{n}\right) \propto$

$$
\sum_{C_{i=1, \ldots, 5}=[S, S a, S q, S r, S v]} \prod_{j=1}^{n} P\left(F_{j} \mid C=c_{i}\right) .
$$

For the SDSS, WISE, and $G_{12}$ data, the computing complex probability is more appropriate because some of the taxonomic classes are very similar in the visible range. Therefore in this research we focus on computing complex probability.

For the a priori probability $P(C)$ we decided to use uniform distribution to allow the inference to be data driven only. Other a priori distributions (such as a frequency prior) could also be used. For example, S- and C-type asteroids are the most common asteroid types, while $\mathrm{Q}$ and $\mathrm{R}$ types are quite rare. To reflect this fact in the inference, one could assume a frequency prior, that is, the $P(C)$ would be equal to the ratio of asteroids of a certain type to the whole population. For example, for S-type asteroids, this would be equal to the ratio of number of $S$ type asteroids to asteroids of all classes. Any frequency prior should stem from an unbiased distribution of taxonomic classes to reflect the real and not biased frequencies.

The conditional probability $P\left(F_{j} \mid C\right)$ for a feature $F_{j}$ can be estimated from the training set of the data as a Gaussian distribution:

$P\left(F_{j} \mid C\right)=\frac{1}{\sqrt{2 \pi \sigma_{F_{j}}}} \exp \left[-\frac{f_{j}-\mu_{F_{j}}}{2 \sigma_{F_{j}}^{2}}\right]$,

where $\mu_{F_{i}}$ and $\sigma_{F_{i}}^{2}$ are the feature-empirical mean and variance, which can be estimated from the training data set or from class templates if known. See the next section for details of obtaining the conditional probabilities. Once all the conditional probabilities $P\left(F_{i} \mid C\right)$ are known, the inference can be made.

Prediction of taxonomic class can be made by computing probabilities for all the Bus-DeMeo classes and selecting the most likely class for an object (Russell et al. 2002):

$\arg \max \left(P\left(C=c_{k}\right) \prod_{j=1}^{n} P\left(F_{j} \mid C=c_{k}\right)\right)_{\text {for } k=1, . ., m}$.

The normalization constant $\alpha$ does not have to be known in advance to make a prediction. It can be computed later as $\left(\sum_{i=1}^{m} P\left(C_{i} \mid F_{1}, \ldots, F_{6}\right)\right)^{-1}$. The general idea is that objects will be classified based on how well they fit into the taxonomic templates of reflectance flux, albedo, and $G_{12} \mathrm{~s}$ for all the classes. The closer the objects features are to the template of some specific class, the higher the probability that the object is of that class. 
Table 1. Average albedo values for the different taxonomic classes.

\begin{tabular}{cccc}
\hline \hline Taxon & $n r$ & Average $p_{V}$ & $\sigma_{p_{V}}$ \\
\hline $\mathrm{Cgh}$ & 14 & 0.062 & 0.043 \\
$\mathrm{~K}$ & 32 & 0.123 & 0.058 \\
$\mathrm{~A}$ & 11 & 0.216 & 0.078 \\
$\mathrm{C}$ & 130 & 0.058 & 0.075 \\
$\mathrm{~B}$ & 56 & 0.069 & 0.097 \\
$\mathrm{D}$ & 26 & 0.09 & 0.155 \\
$\mathrm{Sr}$ & 6 & 0.238 & 0.049 \\
$\mathrm{Sq}$ & 46 & 0.199 & 0.156 \\
$\mathrm{~L}$ & 48 & 0.106 & 0.08 \\
$\mathrm{~S}$ & 370 & 0.21 & 0.088 \\
$\mathrm{~T}$ & 9 & 0.06 & 0.234 \\
$\mathrm{~V}$ & 62 & 0.311 & 0.102 \\
$\mathrm{X}$ & 93 & 0.071 & 0.149 \\
$\mathrm{Ch}$ & 119 & 0.048 & 0.021 \\
$\mathrm{Xc}$ & 50 & 0.073 & 0.161 \\
$\mathrm{Xe}$ & 20 & 0.14 & 0.202 \\
$\mathrm{Cb}$ & 23 & 0.051 & 0.279 \\
\hline
\end{tabular}

Notes. The columns represent: number of objects $(n r)$, average albedo $\left(p_{V}\right)$, and standard deviation $\left(\sigma_{p_{V}}\right)$.

\subsection{Conditional probabilities - training dataset}

Naive classifiers belong to so-called supervised learning methods. Those classifiers can be trained based on expert knowledge or a training dataset. In our approach we combine both learning techniques. Throughout the study we decided to use the Bus-DeMeo taxonomy, which is the most recent commonly used taxonomy; however, for some applications (for example, classification of the E, M, P classes that can only be separated given albedo), other taxonomies might be more appropriate. The classification scheme described in the previous section is also valid for other taxonomies.

To estimate the conditional probabilities of the different classes given albedos, we used the WISE albedo database and the JPL Small-Body Database Search Engine combined with the Planetary Data System (PDS). From the PDS we downloaded a database of asteroids used for defining the Bus-DeMeo Taxonomy (EAR-A-VARGBDET-5BUSDEMEOTAX-1.0). Next we used the JPL tool to extract all the objects with Bus taxonomic class defined. We translated the Bus taxonomy to Bus-DeMeo taxonomy, using the outline scheme provided in DeMeo et al. (2009). In cases where the translation was ambiguous, we assigned more than one class to the object. For example, Ld class in Bus taxonomy corresponds to D or L class in Bus-DeMeo taxonomy, therefore both classes were attributed a new object. We combined both data sets, the PDS and JPL, and then we grouped objects by taxonomic class. Next from the WISE database we selected objects from our list. In the next step we computed mean WISE albedos and standard deviations for each group of asteroids of the same class. Those parameters are listed in Table 1 . The albedo values contributing to the means were weighted by one over their errors. It should be noted that for some taxonomic classes, only a few asteroids are known. There are no albedo estimates for asteroids from classes $\mathrm{O}, \mathrm{Sv}, \mathrm{Q}$, and $\mathrm{Sa}$, therefore those have to be omitted in our classification. With the increasing number of known objects, those values can, however, be refined and increase the overall
Table 2. Average $G_{12}$ values for the different taxonomic classes.

\begin{tabular}{cccc}
\hline \hline Taxon & $n r$ & $G_{12}$ & $\sigma_{G_{12}}$ \\
\hline $\mathrm{Cgh}$ & 20 & 0.615 & 0.228 \\
$\mathrm{~K}$ & 39 & 0.565 & 0.174 \\
$\mathrm{~A}$ & 20 & 0.531 & 0.214 \\
$\mathrm{C}$ & 153 & 0.643 & 0.169 \\
$\mathrm{~B}$ & 65 & 0.639 & 0.182 \\
$\mathrm{D}$ & 35 & 0.543 & 0.209 \\
$\mathrm{Sr}$ & 12 & 0.637 & 0.188 \\
$\mathrm{Sq}$ & 100 & 0.425 & 0.186 \\
$\mathrm{~L}$ & 62 & 1.016 & 0.188 \\
$\mathrm{~S}$ & 564 & 0.341 & 0.163 \\
$\mathrm{~T}$ & 18 & 0.331 & 0.126 \\
$\mathrm{~V}$ & 94 & 0.624 & 0.144 \\
$\mathrm{X}$ & 126 & 0.508 & 0.208 \\
$\mathrm{Ch}$ & 138 & 0.598 & 0.139 \\
$\mathrm{Xc}$ & 70 & 0.368 & 0.223 \\
$\mathrm{Xe}$ & 30 & 0.548 & 0.206 \\
$\mathrm{Cb}$ & 35 & 0.74 & 0.149 \\
\hline
\end{tabular}

Notes. The columns represent number of objects $(n r)$, average phase curve parameter $\left(G_{12}\right)$, and standard deviation $\left(\sigma_{G_{12}}\right)$.

reliability of the method. Though many of the albedo values for the different classes overlap, the albedo data still constitute an important piece of information. For example, asteroids 14419 and 16352 both have very high albedo above 0.31 , suggesting that they might be, for example, of $\mathrm{V}$ type. The general idea is, however, not to infer the possible type of the asteroid based on a single parameter, but instead it is based on a large number of relevant parameters (or features).

For the $G_{12}$ we proceed similarly to albedos by extracting objects with defined taxonomic type and computing mean and standard deviations from the slope parameters database for each taxonomic class (see Table 2 for numerical values). There are no $G_{12}$ estimates for asteroids in $\mathrm{Sa}, \mathrm{Xk}$, and $\mathrm{Sv}$ classes, therefore those also have to be omitted in our classification.

For reflectance fluxes, $\mu_{F_{j}}$ and $\sigma_{F_{j}}$ can be estimated from the spectroscopic templates. Those templates are, for example, available for Bus-DeMeo taxonomic classes via the MIT website. We present the means and standard deviations of reflectance fluxes for the SDSS filters per taxonomic class estimated based on Bus-DeuncertaintyMeo taxonomic templates in Table 3. The taxonomic templates list mean reflectance values and their standard deviations per taxonomic class every $0.05 \mathrm{mi}$ crons. The centers of the SDSS filers bands $(u=0.3543 \mu \mathrm{m}$, $g=0.4770 \mu \mathrm{m}, r=0.6231 \mu \mathrm{m}, i=0.7625 \mu \mathrm{m}, z=0.9134 \mu \mathrm{m})$ lie between the data points provided in the templates, therefore the mean reflectance values for the SDSS filters have to be computed by linear interpolation between the adjacent template points. The corresponding standard deviations for the mean reflectance values in the SDSS filters are also computed accordingly. The $u$ filter reflectance can be computed by a linear extrapolation. However, several of the taxonomic classes show a drop in reflectance in the UV range, and therefore such extrapolation is unreliable and could introduce additional errors. Furthermore, since we are trying to match the data to the Bus-DeMeo classes (which do not extend as far into UV range), including the $u$ filter would just introduce another source of uncertainty. The $u$ filter reflectance values are therefore not used in the classification 
Table 3. Template values of the relative reflectance flux for the SDSS filters.

\begin{tabular}{ccccccccccc}
\hline \hline Type & $F_{u}$ & $\sigma_{F_{u}}$ & $F_{g}$ & $\sigma_{F_{g}}$ & $F_{r}$ & $\sigma_{F_{r}}$ & $F_{i}$ & $\sigma_{F_{r}}$ & $F_{z}$ & $\sigma_{F_{z}}$ \\
\hline Cgh & 0.801 & 0.06 & 0.936 & 0.023 & 1.008 & 0.018 & 1.002 & 0.031 & 1.026 & 0.042 \\
Sq & 0.689 & 0.078 & 0.89 & 0.029 & 1.081 & 0.026 & 1.14 & 0.041 & 1.005 & 0.047 \\
A & 0.494 & 0.065 & 0.811 & 0.025 & 1.152 & 0.019 & 1.279 & 0.029 & 1.128 & 0.047 \\
C & 0.927 & 0.045 & 0.976 & 0.016 & 1.009 & 0.012 & 1.014 & 0.019 & 1.004 & 0.026 \\
B & 0.957 & 0.02 & 0.984 & 0.012 & 1.003 & 0.022 & 0.98 & 0.048 & 0.937 & 0.071 \\
D & 0.81 & 0.082 & 0.93 & 0.031 & 1.062 & 0.025 & 1.176 & 0.065 & 1.292 & 0.11 \\
Sr & 0.638 & 0.205 & 0.88 & 0.048 & 1.087 & 0.025 & 1.161 & 0.049 & 1.005 & 0.055 \\
K & 0.766 & 0.053 & 0.916 & 0.021 & 1.063 & 0.023 & 1.127 & 0.041 & 1.075 & 0.045 \\
L & 0.69 & 0.088 & 0.889 & 0.037 & 1.096 & 0.033 & 1.203 & 0.057 & 1.207 & 0.045 \\
S & 0.68 & 0.071 & 0.884 & 0.027 & 1.091 & 0.027 & 1.185 & 0.049 & 1.081 & 0.059 \\
T & 0.824 & 0.042 & 0.939 & 0.013 & 1.052 & 0.008 & 1.147 & 0.014 & 1.209 & 0.017 \\
V & 0.625 & 0.101 & 0.863 & 0.037 & 1.111 & 0.03 & 1.168 & 0.064 & 0.766 & 0.101 \\
X & 0.898 & 0.051 & 0.967 & 0.019 & 1.022 & 0.007 & 1.076 & 0.019 & 1.118 & 0.033 \\
Ch & 0.911 & 0.041 & 0.975 & 0.014 & 0.988 & 0.009 & 0.981 & 0.018 & 0.994 & 0.029 \\
Cb & 1.019 & 0.03 & 1.006 & 0.011 & 1.0 & 0.007 & 1.017 & 0.006 & 1.025 & 0.031 \\
Xe & 0.843 & 0.03 & 0.931 & 0.033 & 1.067 & 0.032 & 1.116 & 0.037 & 1.124 & 0.049 \\
Xc & 0.868 & 0.067 & 0.956 & 0.018 & 1.029 & 0.006 & 1.074 & 0.007 & 1.096 & 0.005 \\
\hline
\end{tabular}

process. In Table 3 we list the extrapolated reflectance values for the $u$ filter only for reference.

It should be noted that the taxonomic templates for types $\mathrm{O}$, $\mathrm{R}$, and $\mathrm{Cg}$ have very large error envelopes ( $\sigma$ values -0.999 as listed on the MIT webpage). Because the $\mathrm{Cg}$, $\mathrm{O}$, and $\mathrm{R}$ classes were dePned by a single object the standard deviation is set to -0.999 . Therefore those classes are omitted from classification altogether.

Figures 1-3 illustrate the Bus-DeMeo template (shaded area represents the standard deviation for the class) for a V-type asteroid and the interpolated points for the SDSS filters. Any asteroid having SDSS reflectance values fitting the corresponding range of the intra/extrapolated SDSS points (denoted in red in the figure) will have a high V-complex probability and therefore will be classified as a potential V-type candidate. Similar plots can also be made for other taxonomic classes. In these figures we also plot the computed reflectance values for three selected asteroids from the SDSS database. Asteroid 65256 is an outer main belt object, and it was observed by the SDSS twice. Asteroids 14419 and 1652 are inner main belt asteroids, each observed once by the SDSS.

To compare the SDSS against the spectroscopic templates, it is necessary to compute the reflectance values from the raw SDSS photometric data points. To perform this task, we adopt the method by Roig \& Gil-Hutton (2006). First the SDSS color indices have to be corrected by solar colors: $c_{u-r}=(u-r)-1.77$, $c_{g-r}=(g-r)-0.45, c_{r-i}=(r-i)-0.10, c_{r-z}=(r-z)-0.14$. The albedos at each band center, normalized to the albedo at the $r$ band, are then $F_{u}=10^{-0.4 c_{u-r}}, F_{g}=10^{-0.4 c_{g-r}}, F_{i}=$ $10^{0.4 c_{r-i}}, F_{z}=10^{0.4 c_{r-z}}$. The errors can be estimated as $\Delta F / F=$ $0.9219 \Delta c(1+0.4605 \Delta c)$, where $\Delta c$ are color errors computed as the usual root squared sum of the corresponding magnitude errors. For more details please see Roig \& Gil-Hutton (2006). The computed flux values have to then be slid up or down to the template. This is done by aligning the 0.55 microns normalization point of the template and the data.

Two things should be noted: i) given a different set of asteroids in each taxonomic complex can result in slightly different means and variances for albedo and G12 parameter; ii) with increasing number of taxonomic classifications, those parameters will be refined better and also correlations between the different features will be better known.

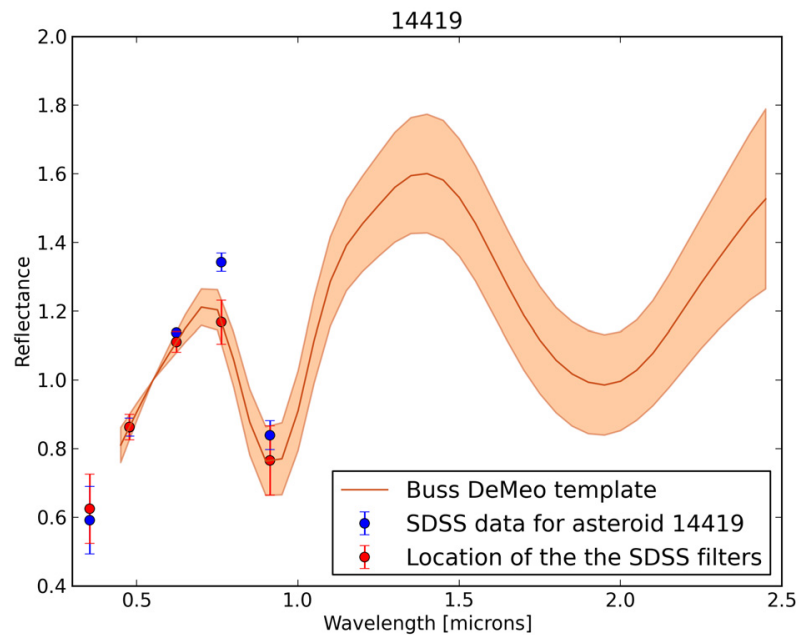

Fig. 1. SDSS data points for asteroid (14419) 1991 RK23 as compared to the Bus-DeMeo V-class template. The shaded area denotes the standard deviation of the template. The $u$ filter reflectance is added as an linearly extrapolated point for reference only.

\subsection{Including uncertainties}

For better probability estimation there is an option of including the features uncertainties in the probability computation. Each measurement is fraut with both systematic and random errors (for example, some of the SDSS measurements show large photometric errors). Consequently the real value of a feature can lie anywhere in the given uncertainty region. Each feature can therefore be represented by a large number of possible values. Each of the features is sampled within its uncertainty. Next classification as described in the section above is performed. This results in multiple classifications (and probabilities $p(c \mid f)$ for a specific class $c$ and specific sampled set of features $f$ ) per single object. Objects having small feature uncertainties will often be categorized in the same class. Those with large feature uncertainties will be categorized to many different classes. The final decision classifying the object can be made based on how many times the object was classified into which category. The final probability for a given class $\mathrm{C}$, including the uncertainties, can then be 


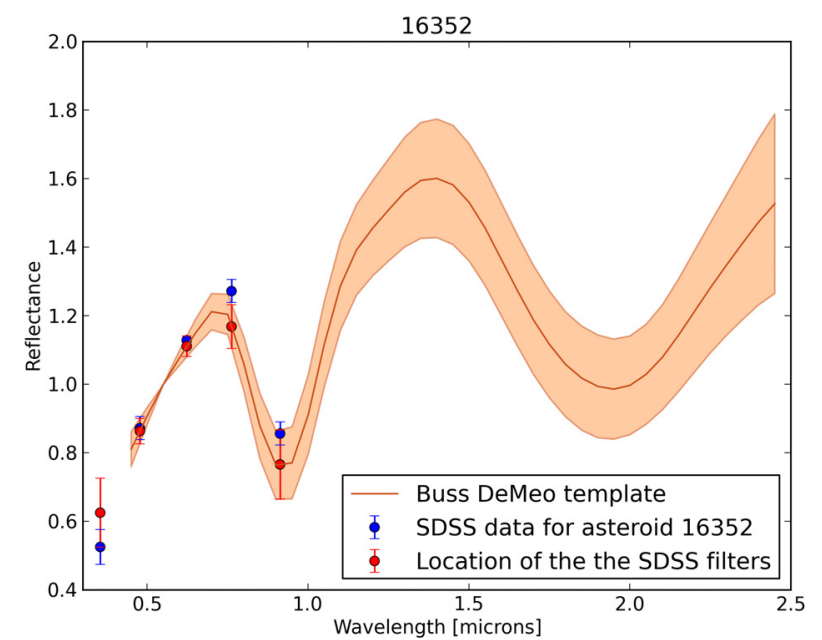

Fig. 2. SDSS data points for asteroid (16352) $1974 \mathrm{FF}$ as compared to the Bus-DeMeo V-class template. The shaded area denotes the standard deviation of the template. The $u$ filter reflectance is added as an linearly extrapolated point for reference only.

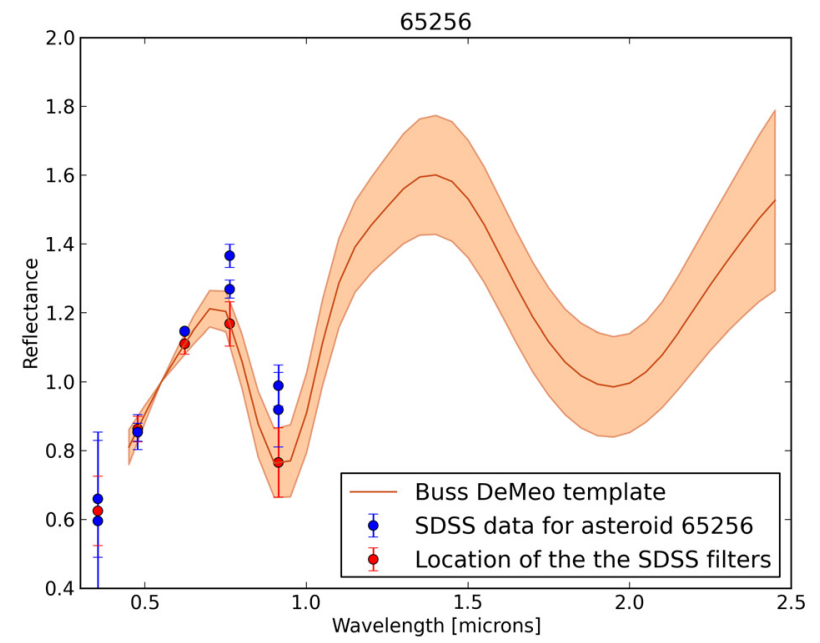

Fig. 3. SDSS data points for asteroid (65256) 2002 FP34 as compared to the Bus-DeMeo V-class template. The shaded area denotes the standard deviation of the template. The $u$ filter reflectance is added as an linearly extrapolated point for reference only.

computed as

$P(C)=\int_{F_{\mathrm{L}}}^{F_{\mathrm{U}}} p(C \mid \mathbf{F}) \mathrm{d} \mathbf{F}$,

where $\mathbf{F}$ is a set of features and $F_{\mathrm{U}}, F_{\mathrm{L}}$ are the upper and lower boundary values obtained from features uncertainties. In practice, the taxonomic class probability can be computed as a weighted fraction of class classifications as compared to all classes:

$P(C)=\frac{\sum_{j=1}^{N_{C}} w_{j}}{\sum_{k=1}^{N_{\text {all }}} w_{k}}$,

where $N_{C}$ is the number of times an object was classified as a given taxonomic class and $N_{\text {all }}$ is total number of classifications. The $w_{j}$ and $w_{k}$ weights are the probability values $p(c \mid f)$ computed for the class under consideration and all the other classes respectively.

\subsection{Comparison with other methods and validation}

To compare our method with those already available in the literature, we performed classification of basaltic candidates indicated in Moskovitz et al. (2008a), Roig \& Gil-Hutton (2006) and Solontoi et al. (2012). In particular, we compared the classification for all the numbered asteroids in the candidate lists that have all the features available (colors, albedo, $G_{12} \mathrm{~s}$ ). Additionally, we checked how many of those objects were observed spectrally and confirmed V-type. In Table A.1 we list the classification and probabilities for those objects. In most of the cases probabilities based on different sets of observations agree with each other. When few datasets were available for object (for example asteroid observed a few times by the SDSS) we have listed the highest probabilty of V-type or highest probabilty of any other class if no classification ended as V-type.

Out of 50 candidates listed by Moskovitz et al. (2008a), nine numbered objects have all the features available. Out of those all, but two ((55092) 2001 QO123 and, (50802) 2000 FH27) were classified as V-type candidates using our method. Object (50802) $2000 \mathrm{FH} 27$ has been observed twice by the SDSS and the two datasets give completely different classifications $(96 \%$ D-type or 57\% Sr-type). While (55092) 2001 QO123 produces a better fit to Sr-type in our method. It is worth noticing that those objects were also not identified by Roig \& Gil-Hutton (2006) as V-type candidates. Spectroscopic observations and classification is available for seven objects from this list. Asteroid (46690) 1997 AN23 was classified as S-type object after spectroscopic observations (Moskovitz et al. 2008a) even though it was listed as a V-type candidate by all the authors. The object also had $72 \%$ probability of beeing V-type in our method. It is worth noticing that the object was observed by the SDSS four times. The classification based on the other observations in our method leads to S-complex (Sr type) classification three out of four times (with probabilities of 52\%, 52\%, 64\%). Spectroscopic observations are also available for six other objects from this list. All of those were classified as V type based on spectra. All of those were also classified as V-type candidates in our method. In general, our method is in good agreement with that of Moskovitz et al. (2008a) (77\% coverage among the candidates) and spectroscopic observations for those objects.

Out of 233 V-types candidates that are members of the Vesta family indicated by Roig \& Gil-Hutton (2006), there are 71 numbered asteroids that have all features available. Out of those all but three objects ((20437) 1999 JH1, (10157) Asagiri, (16452) Goldfinger) were classified as V-type candidates using our method. Out of 266 V-types candidates that are not members of the Vesta family indicated by Roig \& Gil-Hutton (2006), 78 numbered objects have all the features available. Out of those, all but two ((44496) 1998 XM5 and, (10666) Feldberg) were also classified as V type using our method. The objects with nonmatching classification are mostly classified as $\mathrm{Sq}$ or $\mathrm{Sr}$ in our method. The S-complex classifications agree between the different SDSS observation sets. In general, 144 objects (out of 149) indicated by Roig \& Gil-Hutton (2006) were also classified as V-type candidates in our method. Out of those objects 17 were spectrally observed and confirmed V types. All of those $17 \mathrm{ob}-$ jects were classified as V-type candidates by our method. There is good agreement of our method with Roig \& Gil-Hutton (2006) (96\% candidate coverage among the non-vestoids and vestoids) and spectroscopic observations.

Out of 2023 numbered objects fitting the AVAST selection criteria $(i-z<-0.2), 673$ have all the features available, and 25 have been observed spectrally Solontoi et al. (2012). All 
Table 4. Asteroids selected for observations.

\begin{tabular}{|c|c|c|c|c|c|}
\hline $\begin{array}{c}\text { Asteroid } \\
\text { number }\end{array}$ & $\begin{array}{l}\text { Location } \\
\text { in MB }\end{array}$ & albedo & $i-z$ & $G_{12}$ & Prob \\
\hline 15551 & outer & $0.28 \pm 0.03$ & $-0.15^{ \pm 0.04}$ & $0.50_{-016}^{+0.16}$ & S $0.72(0.01)$ \\
\hline 65256 & outer & & $-0.23^{ \pm 0.08}$ & $0.80^{+\infty}$ & А $0.79(0.1)$ \\
\hline 1979 & inner & $0.39^{ \pm 0.04}$ & $-0.21 \pm 0.04$ & $0.02_{-0.1}^{+0.1}$ & Sr $0.43(0.04)$ \\
\hline 10484 & inner & $0.23^{ \pm 0.06}$ & $-0.23^{ \pm 0.03}$ & $0.51_{-0.1}^{+0.1}$ & Sr $0.43(0.18)$ \\
\hline 41485 & inner & & $-0.44^{ \pm 0.03}$ & $0.47_{-0.1}^{+0.1}$ & V 0.67 \\
\hline 13679 & inner & $0.31^{ \pm 0.03}$ & $-0.12^{ \pm 0.03}$ & $0.61_{-0.15}^{+0.17}$ & A $0.59(0.01)$ \\
\hline 18775 & inner & & $-0.17 \pm 0.05$ & $0.74+0.13$ & A $0.37(0.26)$ \\
\hline 30243 & inner & & $-0.25^{ \pm 0.06}$ & $0.50_{-0.20}^{+0.14}$ & V 0.49 \\
\hline 40521 & mid & $0.28^{ \pm 0.03}$ & $-0.32 \pm 0.07$ & $0.39^{-0.17}$ & V $0.89(0.89)$ \\
\hline 31455 & mid & & $-0.21^{ \pm 0.07}$ & $0.50_{-0.16}^{+0.17}$ & L $0.38(0.26)$ \\
\hline 15717 & mid & & $-0.07 \pm 0.16$ & $0.60_{-0.12}^{+0.12}$ & V 0.49 \\
\hline 33493 & mid & & $-0.13^{ \pm 0.04}$ & $0.44_{-0.12}^{-0.12}$ & L $0.41(0.16)$ \\
\hline 11699 & inner & $0.23^{ \pm 0.06}$ & $-0.28^{ \pm 0.04}$ & $0.36_{-0.11}^{+0.12}$ & Sr $0.31(0.18)$ \\
\hline 14419 & inner & $0.32^{ \pm 0.04}$ & $-0.47^{ \pm 0.06}$ & $0.45_{-0.12}^{+0.12}$ & V 1.0 \\
\hline 16352 & inner & $0.37^{ \pm 0.04}$ & $-0.39^{ \pm 0.06}$ & $0.51_{-0.13}^{+0.13}$ & V 0.99 (0.99) \\
\hline
\end{tabular}

of the observed objects turned out to be $\mathrm{V}$ types except for 46262 (S-type) and 27202 (A-type). Two other objects were misclassified by our method, namely 20455 and 32272, for which the spectroscopic observations are indicative of $\mathrm{V}$ type, but the classification places them to be S-complex objects. Overall, our method predicted the right complex for 21 out of 25 objects observed and is in good agreement with the spectroscopic observations. However a large number of V-type candidates fitting the AVAST criteria are classified as S- C- or X- complex objects in our method, though it should be noted that only two object from the non-matching candidate list (32272 and 20455) were observed.

Overall, the method correctly classified most of the spectrally confirmed objects and shows good agreement in candidate selection with the methods by Moskovitz et al. (2008a) and Roig $\&$ Gil-Hutton (2006). There is less overlap with the basaltic candidates indicated by Solontoi et al. (2012) though. Our method predicted the taxonomic complex correctly for $88 \%$ of the objects observed listed in Table A.1.

\subsection{V-type candidates}

We have predicted taxonomic complexes for all the asteroids present in the given datasets. We examine asteroids for which all the features (SDSS photometry, WISE albedo, and $G_{12}$ ) are available. In Fig. 4 we present the distribution of V-type candidates as predicted by our method. Most of them are clustered around the Vesta family, but also scattered in the mid and outer main belt. Those are objects that were classified as V-type candidates at least once (based on one set of observations). Other than the Vesta family, there seems to be no clustering of candidates around other families. In Table 6 we list our candidates located in mid and outer main belt. Though the list is extensive, most of the candidates have SDSS data uncertainties $>10 \%$ or classifications based on different datasets disagree with each other, therefore the classification should be taken with caution. Our top candidates (classification based on data with smaller uncertainties) include 11 first asteroids listed in Table 6. Out of those (21238) Panarea has already been confirmed as a V-type asteroid (Binzel et al. 2006; Hammergren et al. 2006; Roig et al. 2008). Asteroid (10537) 1991 RY16 is an R-type object (also originating in a differentiated body) previously observed by Moskovitz et al. (2008b), and 40521 (1999 RL95) was

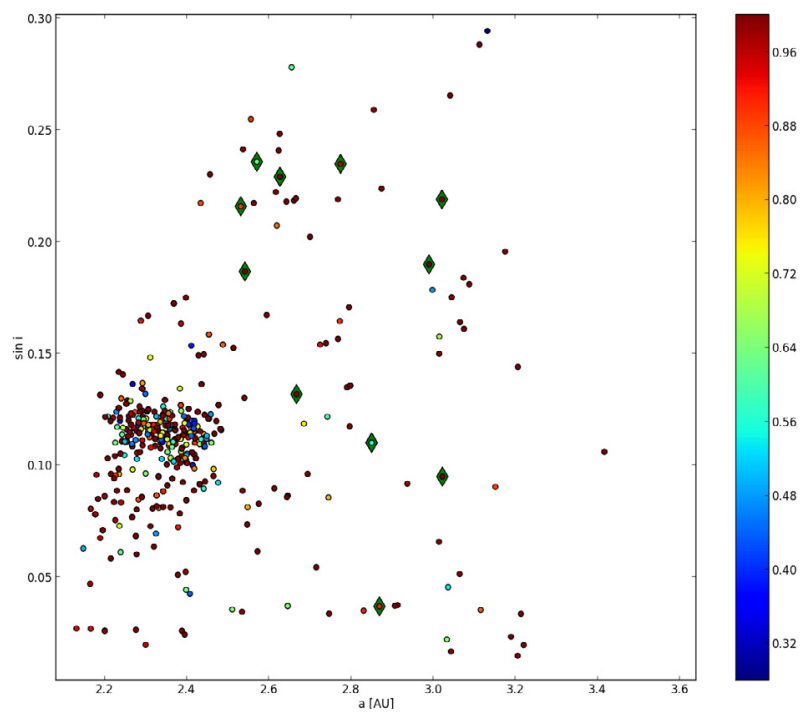

Fig. 4. Distribution of V-type candidates in the main belt. Color corresponds to probability. Candidates with good quality data are additionally denoted with a diamond marker.

previously indicated as a basaltic candidate and also observed Roig et al. (2008).

\section{Observations and data reduction}

We selected a number of objects to be observed, as listed in Table 4. We focus on objects that are located in the mid and outer main belt, as well as in the inner main belt, but outside the Vesta dynamical family (definition of Vesta family extracted from HCM Asteroid Families V2.0. EAR-A-VARGBDET-5NESVORNYFAM-V2.0. NASA Planetary Data System, 2012). We have avoided candidates that were targeted by other V-type candidate surveys.

The observations were performed in 2012 at two different telescopes: the Nordic Optical Telescope (NOT), and the South African Large Telescope (SALT). NOT is located in the Canary Islands and SALT in South Africa.

At the $2.56 \mathrm{~m}$ NOT, we used the Andalucia Faint Object Spectrograph and Camera (ALFOSC) combined with lowresolution grism number 11 and slit $1.8^{\prime \prime}$ for all of our targets 


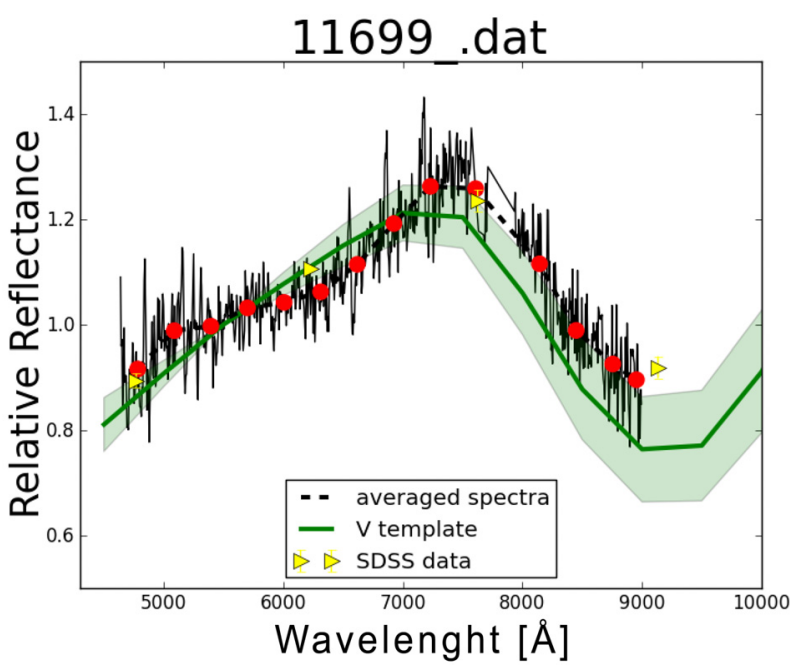

Fig. 5. Spectra of asteroid (11699) 1998 FL105 obtained at the SALT. The triangles denote the reflectance values computed from the SDSS photometry. The thick line represents the taxonomical template for the V-type, and the shaded area is the standard deviation of the template.

except for one. For asteroid (15551) Paddock, we used grism 12 with $2.5^{\prime \prime}$ slit since the object was observed during a test night for which Grism 11 was not available. Grism 11 gives wavelength coverage from 0.39 microns to 0.995 microns and has a dispersion of $4.8 \AA$ per pixel. Grism 12 gives wavelength coverage 0.51 microns to 1.1 microns and has a dispersion of $13 \AA$ per pixel. Grism 12 has a build-in blocking filter OG515. No other blocking filters were used. Differential tracing was employed.

At the $10 \mathrm{~m}$ SALT telescope (Buckley et al. 2006) we used the Robert Stobie Spectrograph (RSS; Burgh et al. 2003; Kobulnicky et al. 2003). The 4" slit was imaged through the pc04600 order-blocking filter onto the pg0300 grating, which resulted in the FWHM resolution $\Delta \lambda=46 \AA(R \simeq 150)$. The spectrum was recorded with the mosaic CCD camera in the useful range from $4660 \AA$ to $9000 \AA$, with a gap (due to a physical gap between the three CCD segments) from $7746 \AA$ to $7917 \AA$. In this setup the reciprocal dispersion at the center of the spectrum was $1.5 \AA$ per pixel, therefore we used the $4 \times 4$ bining, which gave us seven superpixels per FWHM resolution.

At the time of observations, the non-sidereal tracking of the telescope had not been commissioned yet, so we oriented the spectrograph slit along the asteroid trail and took 16 consecutive exposures of $120 \mathrm{~s}$. During the exposure time, the sky movement of each of the observed asteroids was equal to the seeing, the FWHM of which was 1'.2-1'.4. This procedure worked as a natural dithering, which helped to average the fringing pattern out at the red end of the spectra. It should be noted that thanks to the SALT Atmospheric Dispersion Compensator and a wide slit, the influence of the differential refraction was minimized. For each asteroid a solar-analog star was observed at a similar airmass.

Primary reduction of the data was done on-site with the SALT science pipeline (Crawford et al. 2010). After that, the bias- and gain-corrected and mosaicked long-slit spectra were reduced in a standard way with the IRAF package. The spectrum of Ar lamp was used to calibrate the wavelength scale, and spectral flats to correct for pixel-to-pixel variations. After mediancombining all individual spectra of a given asteroid, the obtained spectrum was divided by the spectrum of the solar-analog star.

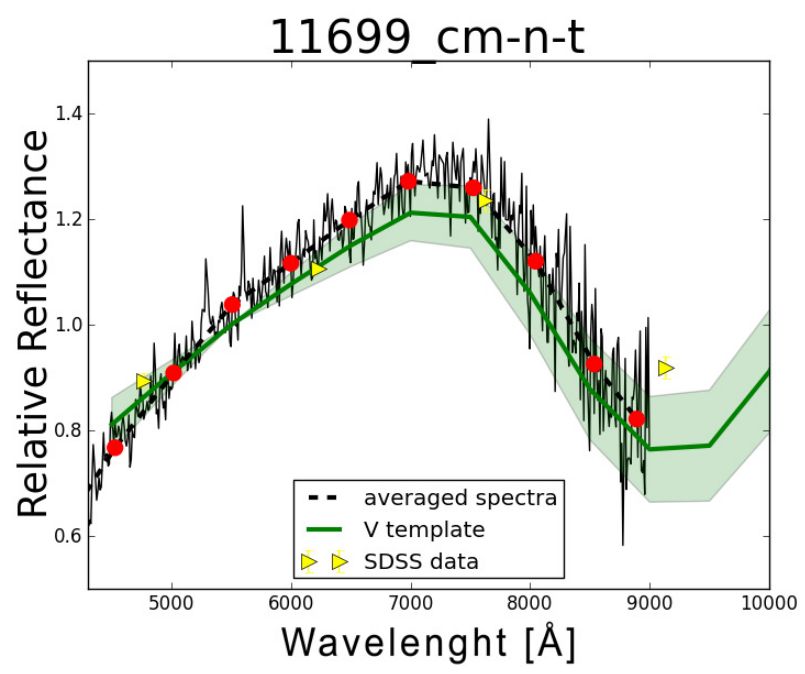

Fig. 6. Spectra of asteroid (11699) 1998 FL105 obtained at the NOT. The triangles denote the reflectance values computed from the SDSS photometry. The thick line represents the taxonomical template for the V-type, and the shaded area is the standard deviation of the template.

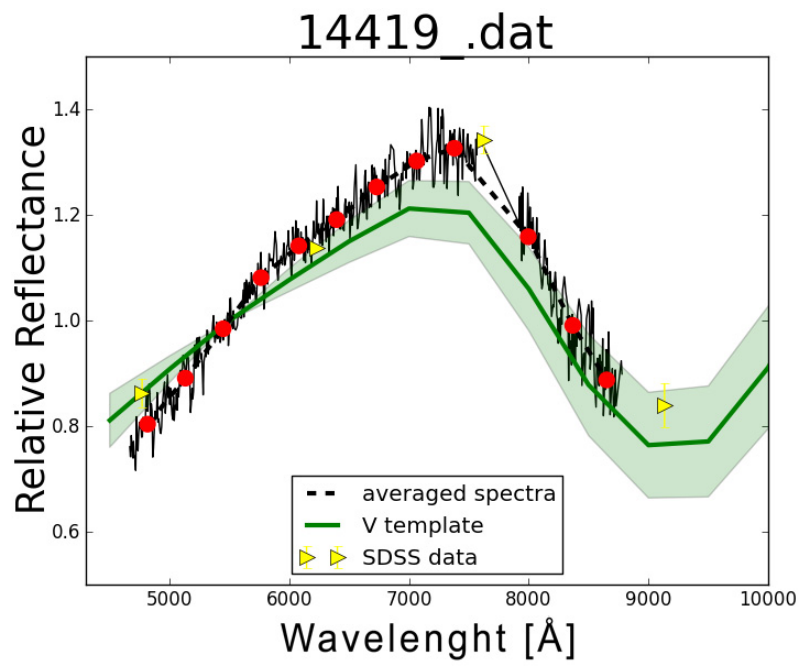

Fig. 7. Same as in Fig. 5 for asteroid (14419) 1991 RK23.

\section{Results}

We present the obtained spectra in Figs. 5 to 20 . In Table 5 we present the observing circumstances along with the assigned taxonomic classification. The preliminary classification is determined by how closely the asteroid spectrum is fitted by the standard spectrum of each class using a curve matching algorithm. This involves fitting the spectrum with a polynomial curve and comparing this curve to the standard spectrum at the wavelength given in the taxonomy. The class producing the smallest standard deviation is then selected. The reliability is based on the number of points used for the comparison with the taxonomic templates. For more details please see Popescu et al. (2012). For most of the objects we observed two solar analogs, one before taking the science exposure and the second after the science exposure. The spectra of asteroids were divided by both solar analogs and then compared to each other. Asteroids were assigned preliminary taxonomical classes ${ }^{1}$.

1 http://m4ast.imcce.fr/ 
Table 5. Observing circumstances and classification.

\begin{tabular}{cccccccccc}
\hline \hline $\begin{array}{c}\text { Asteroid } \\
\text { number }\end{array}$ & Telescope & UT date & $\begin{array}{c}\text { Phase } \\
\text { angle }\end{array}$ & $V$ mag & $\begin{array}{c}\text { Air } \\
\text { mass }\end{array}$ & $\begin{array}{c}\text { Solar } \\
\text { analog }\end{array}$ & $\begin{array}{c}\text { Preliminary } \\
\text { class }\end{array}$ & $\begin{array}{c}\text { Std. dev. } \\
\text { Reliability } \\
\text { of classification }\end{array}$ \\
\hline 15551 & NOT & $02-01-2013$ & $19^{\circ}$ & 18.6 & 1.2 & 51 Peg & Q & 0.042 & $85.3 \%$ \\
65256 & NOT & $07-05-2013$ & $3^{\circ}$ & 18.8 & 1.5 & $\begin{array}{c}107-998 \\
0.062(\mathrm{~A})\end{array}$ & $24.3 \%$ \\
1979 & NOT & $07-05-2013$ & $25^{\circ}$ & 18.2 & 1.2 & $102-1081$, HD 101364 & A & 0.279 & $26.8 \%$ \\
10484 & NOT & $07-05-2013$ & $18^{\circ}$ & 17.7 & 1.3 & HD 101364, HD 144873 & V & 0.132 & $29.2 \%$ \\
41485 & NOT & $07-05-2013$ & $3^{\circ}$ & 18.8 & 1.4 & $107-689,107-998$ & V & 0.077 & $21.9 \%$ \\
13679 & NOT & $07-05-2013$ & $14^{\circ}$ & 16.5 & 1.5 & $112-1333$ & S-complex & $0.023(\mathrm{~S})$ & $24.3 \%$ \\
18775 & NOT & $07-05-2013$ & $19^{\circ}$ & 18.5 & 1.4 & $107-998$, HD 144873 & S-complex & $0.05(\mathrm{Sr})$ & $29.2 \%$ \\
30243 & NOT & $08-05-2013$ & $5^{\circ}$ & 18.2 & 1.6 & HD 147284, 110-361 & S-complex & $0.04(\mathrm{Sq})$ & $29.2 \%$ \\
40521 & NOT & $11-05-2013$ & $20^{\circ}$ & 20.1 & 1.8 & HD 147284, HD 144873 & V & 0.102 & $29.2 \%$ \\
31455 & NOT & $11-05-2013$ & $13^{\circ}$ & 18.5 & 1.2 & HD 144873, 102-1081 & S-complex & $0.030(\mathrm{Sq})$ & $29.2 \%$ \\
15717 & NOT & $11-05-2013$ & $18^{\circ}$ & 18.6 & 1.3 & $112-1333$, HD 147284 & A & 0.049 & $29.2 \%$ \\
33493 & NOT & $11-05-2013$ & $18^{\circ}$ & 18.0 & 1.5 & $112-1333$ & S-complex & $0.020(\mathrm{Sa})$ & $21.9 \%$ \\
11699 & NOT & $03-07-2013$ & $27^{\circ}$ & 17.3 & 1.6 & HD 148642, 102-1081 & V & 0.026 & $24.3 \%$ \\
14419 & SALT & $21-03-2013$ & $6^{\circ}$ & 17.5 & 1.2 & $107-998,107-684$ & V & 0.060 & $19.5 \%$ \\
16352 & SALT & $22-04-2013$ & $21^{\circ}$ & 17.6 & 1.2 & $107-684$ & V & 0.018 & $21.9 \%$ \\
11699 & SALT & $26-04-2013$ & $10^{\circ}$ & 15.8 & 1.2 & $102-1081$ & - & - \\
\hline
\end{tabular}

Notes. Asteroids were classified using the M4AST tool (Popescu et al. 2012).

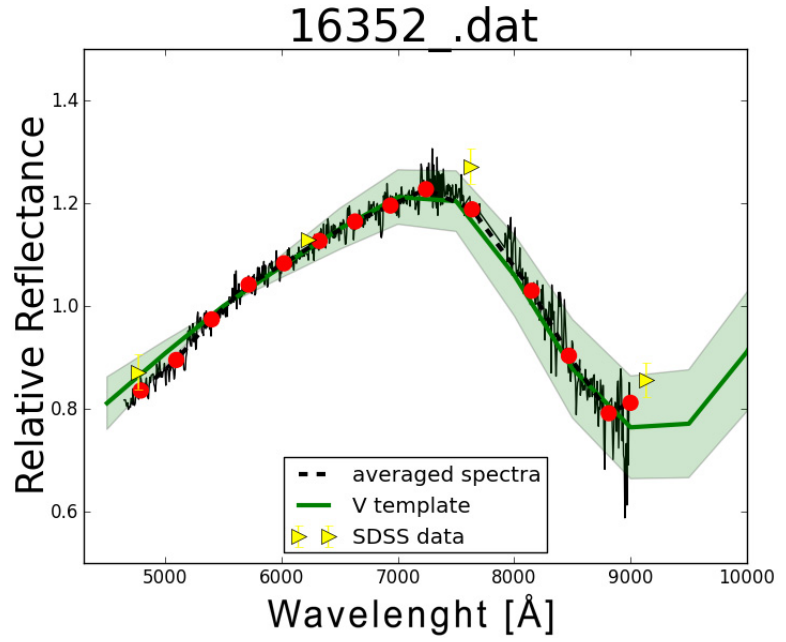

Fig. 8. Same as in Fig. 5 for (16352) 1974 FF.

We targeted several inner main belt asteroids. Out of those (11699) $1998 \mathrm{FL}_{105}$, (14419) $1991 \mathrm{RK} 23$, (41485) $2000 \mathrm{QF}_{51}$, and 16352 (1974 FF) are V types. Asteroid 11699 was observed twice. First at the SALT and then because of a peculiar absorption around 0.65 microns, the observations were repeated at the NOT. Second observations showed no absorption band around 0.65 microns. Therefore we conclude that the feature is most likely due to weather or instrumental causes. Asteroids (1979) Sakharov and (10484) Hecht are basaltic pairs from the inner main belt that we targeted for a different observing program (Polishook et al. 2013) during our run. Three other inner main belt asteroids, (13679) Shinanogawa, (18775) Donaldeng and, (39243) $2000 \mathrm{YU}_{76}$ are from the S-complex. Asteroid (40521) 1999 RL95 was already identified as basaltic by Roig et al. (2008). Based on our spectra we also assign a V type to this object. In the outer main belt, we found several objects that are worth investigating in the NIR. Particularly asteroids (65256) $2002 \mathrm{FP}_{34}$, (15551) Paddock, and (15717) 1990 BL1 have deep absorption bands. All those mid-to-outer main belt objects are especially interesting for investigating the "missing dunite problem" Burbine et al. (1996). If confirmed in NIR, those object

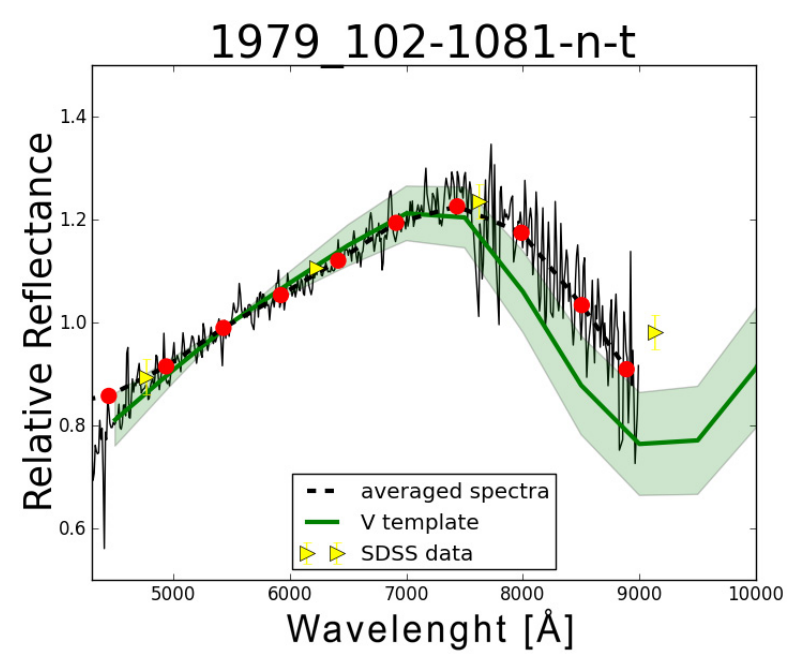

Fig. 9. Same as in Fig. 6 for asteroid (1979) Sakharov.

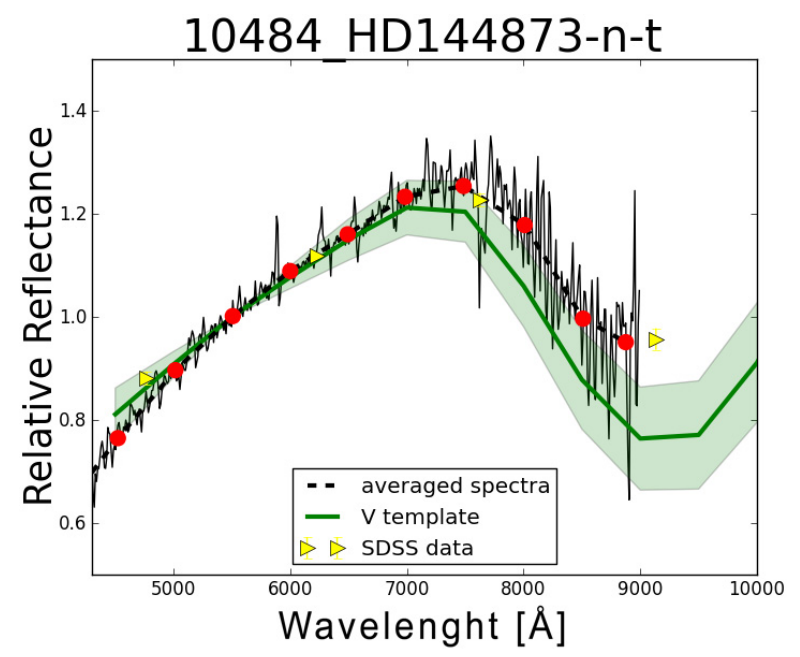

Fig. 10. Same as in Fig. 6 for asteroid (10484) Hecht.

could contribute to the modest inventory of outer main belt objects of possible differentiated origins. For (15551) Paddock, 


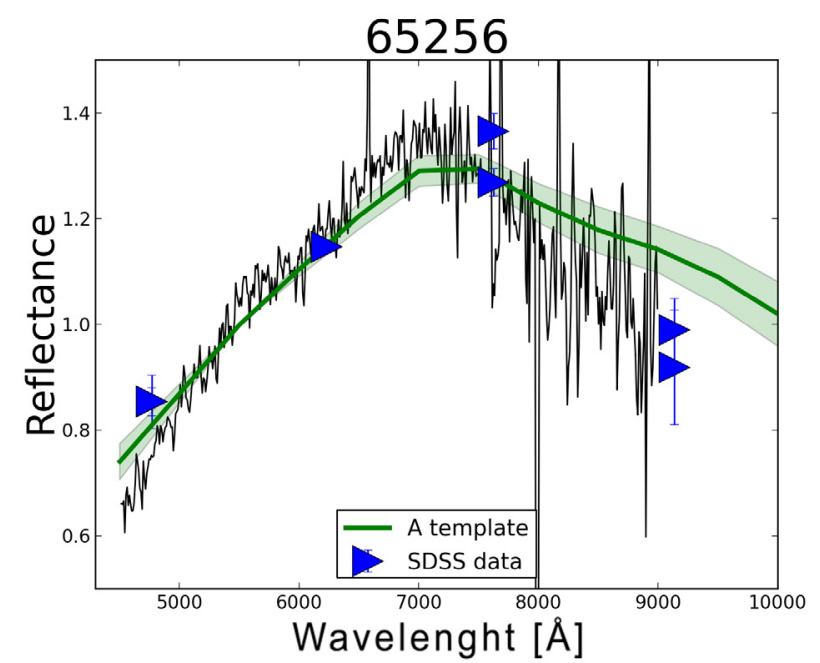

Fig. 11. Spectra of asteroid (65256) 2002 FP34 obtained at the NOT. The triangles denote the reflectance values computed from the SDSS photometry. The thick line represents the taxonomical template for the A-type, and the shaded area is the standard deviation of the template.

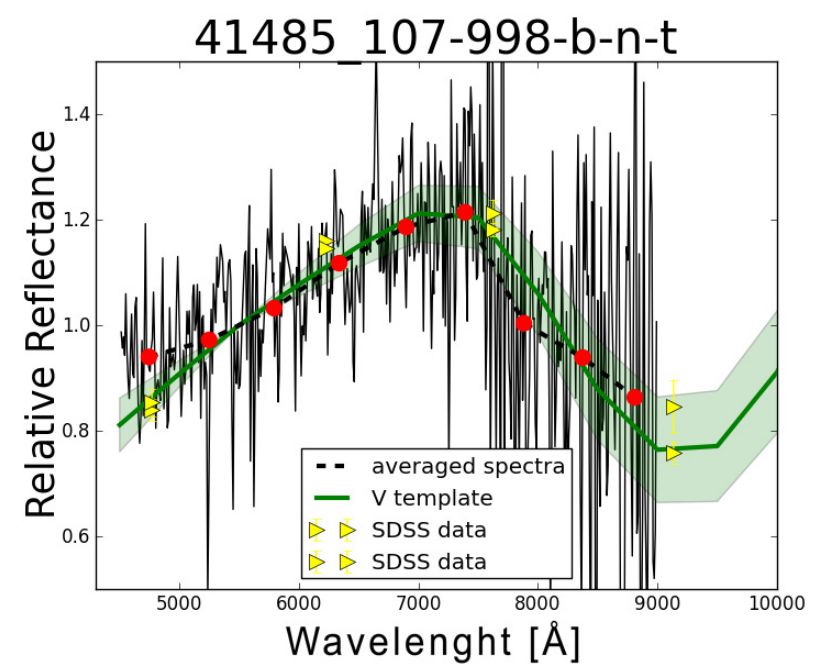

Fig. 12. Same as in Fig. 6 for asteroid (41485) 2000 QF51.

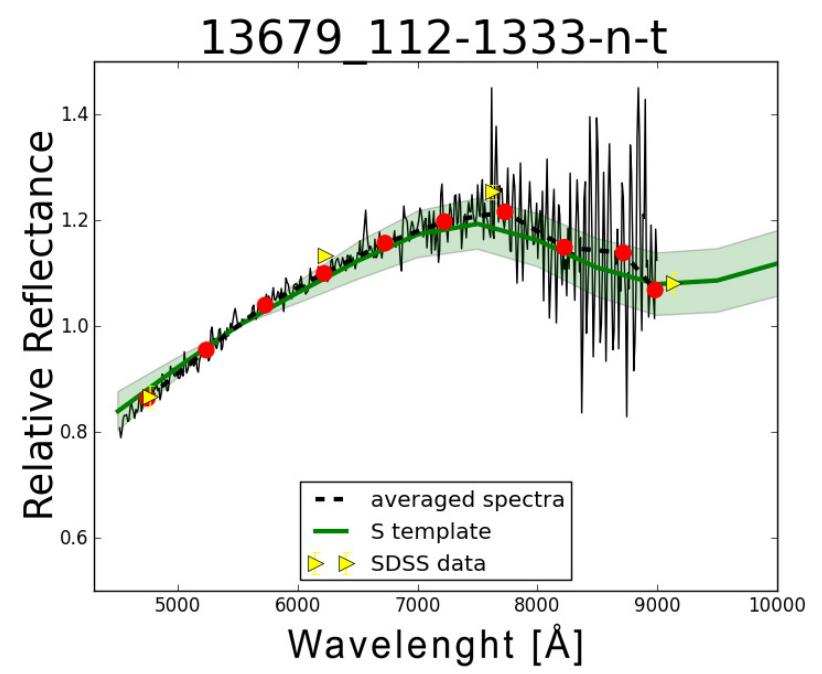

Fig. 13. Spectra of asteroid (13679) Shinanogawa. The triangles denote the reflectance values computed from the SDSS photometry. The thick line represents the taxonomical template for the S-type, and the shaded area is the standard deviation of the template.

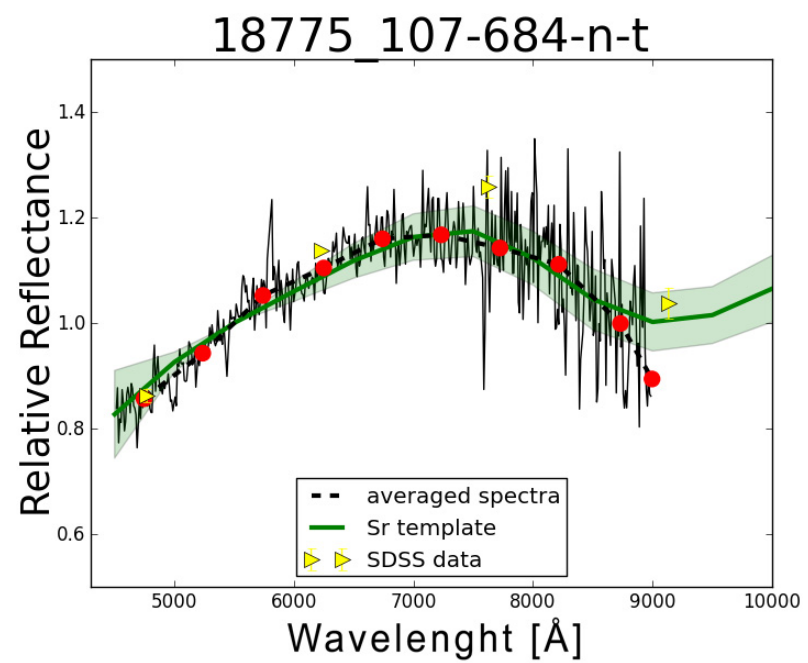

Fig. 14. Spectra of asteroid (18775) Donaldeng. The triangles denote the reflectance values computed from the SDSS photometry. The thick line represents the taxonomical template for the Sr-type, and the shaded area is the standard deviation of the template.

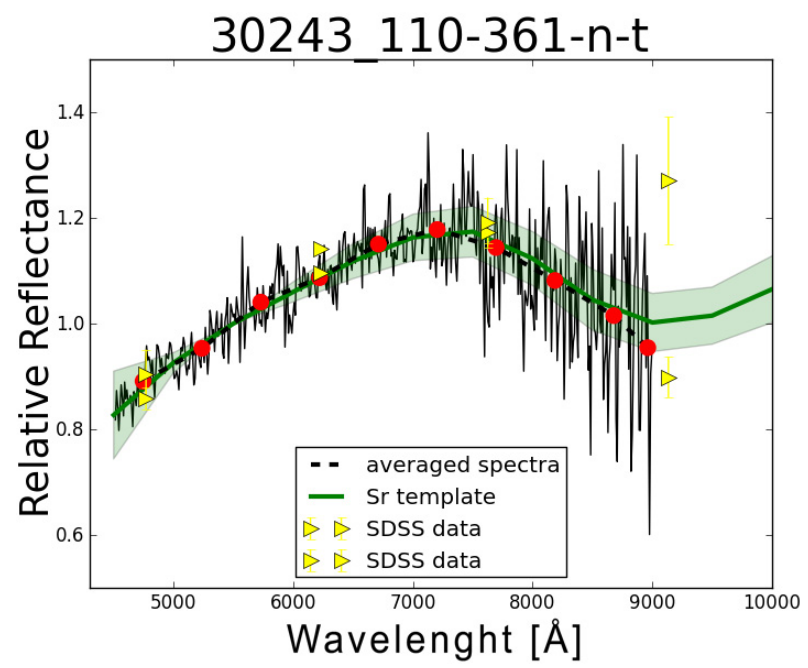

Fig. 15. Spectra of asteroid (30243) 2000 HS9. The triangles denote the reflectance values computed from the SDSS photometry. The thick line represents the taxonomical template for the Sq-type, and the shaded area is the standard deviation of the template.

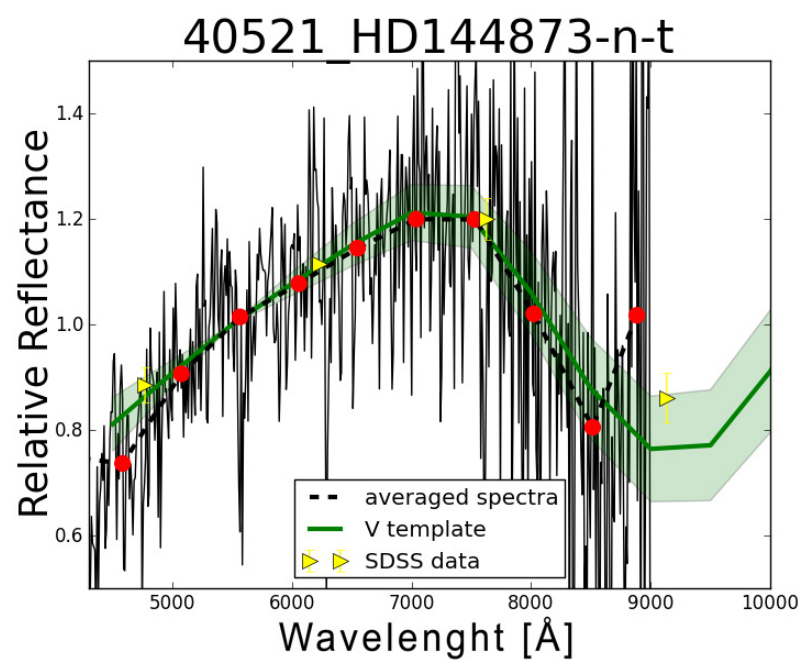

Fig. 16. Same as in Fig. 6 for asteroid (40521) 1999 RL95. 


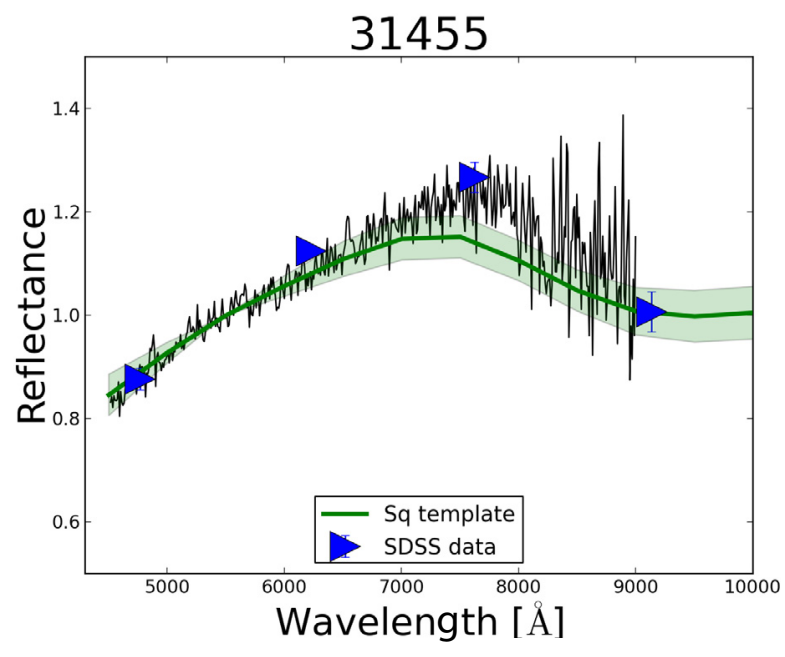

Fig. 17. Same as in Fig. 15 for asteroid 31455.

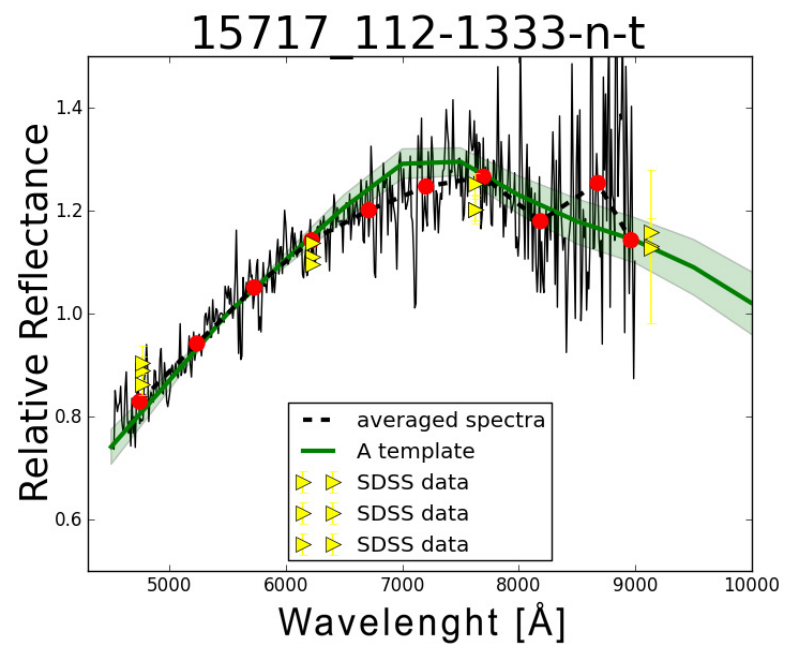

Fig. 18. Same as in Fig. 11 for asteroid (15717) 1990 BL1.

we have combined our data with NIR from the IRTF (courtesy of Francesca DeMeo) and classified it as an Q-type object. However it should be noted that the visible spectrum resembles a V-complex object more and the NIR resembles an S-complex object. It should also be mentioned that owing to shorter wavelength coverage of grism 12 , the normalization at 0.55 microns had to be done by extrapolating the spectra to that region, therefore leading to a less reliable classification.

\section{Conclusions}

We have developed a new selection method. The method uses the SDSS photometric data, WISE albedos, and $G_{12}$ s to predict taxonomic type of asteroids. The method can be improved further when new objects are spectrally observed and classified. New classifications expand the training set and narrow the conditional probabilities. The efficiency of the method is lower than other methods, partly because we allow noisy data to enter our classification. This procedure is purposeful. Even though the SDSS MOC has already been exploited, we show that there is still room for new discoveries within this catalog when merged with other large databases. For example targeting objects from the WISE database having high-albedo can result in new discoveries of high albedo taxonomic types, such as the V type.

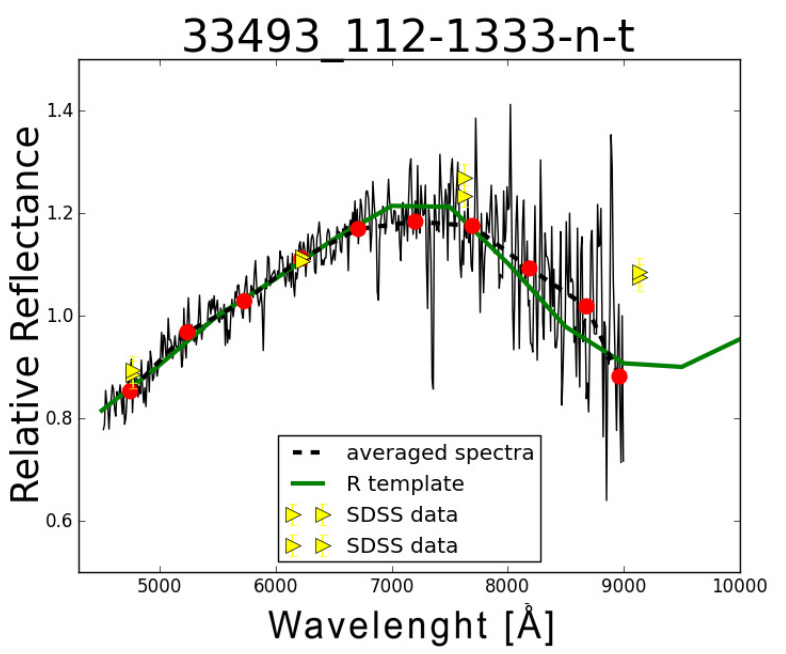

Fig. 19. Spectra of asteroid 33493. The triangles denote the reflectance values computed from the SDSS photometry. The thick line represents the taxonomical template for the Sa-type, and the shaded area is the standard deviation of the template.

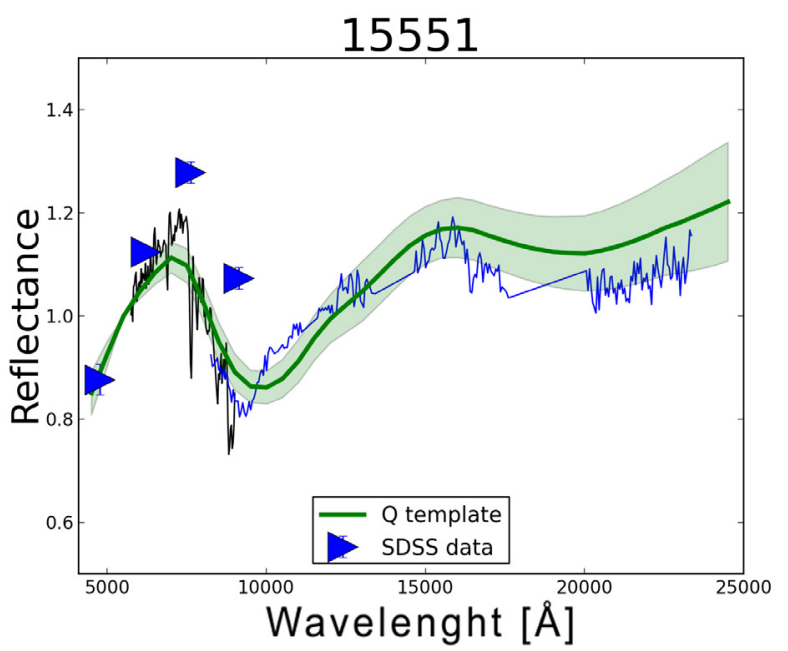

Fig. 20. Spectra of asteroid (15551) Paddock obtained at the NOT and IRTF (courtesy of Francesca DeMeo). The triangles denote the reflectance values computed from the SDSS photometry. The thick line represents the taxonomical template for the Q-type, and the shaded area is the standard deviation of the template.

We found several $\mathrm{V}$ type asteroids, most of them in the inner main belt. Three asteroids in the mid and outer main belt look especially interesting: 65256, 15551, 15717. All of them show deep absorption bands near 1.0 micron and should be further investigated in the NIR. Additionally, we also plan to target our strong V-type candidates during their nearest opposition.

The distribution of V-type candidates shows a clustering around the Vesta family and scatter in the mid-to-outer main belt. An in other studies, we find that V-types candidates are not plentiful beyond 2.5 AU. Given the increasing spectroscopic reach of the modern telescopes, we believe that dynamical, physical, and geological paths should be explored in solving the missing dunite problem.

Acknowledgements. D.O. was supported by the Polish National Science Center, grant number 2012/04/S/ST9/00022. T.K. was supported by the Polish National Science Center, grant number N 203 403739. The work was based on observations made with the Nordic Optical Telescope, operated by the Nordic 
Optical Telescope Scientific Association at the Observatorio del Roque de los Muchachos, La Palma, Spain, of the Instituto de Astrofisica de Canarias and observations made with the South African Large Telescope at the South African Astronomical Observatory, Sutherland, South Africa.

\section{References}

Ammannito, E., De Sanctis, M. C., Palomba, E., et al. 2013, Nature, 504, 122 Barrat, J.-A., Yamaguchi, A., Zanda, B., et al. 2010, Geochim. Cosmochim. Acta, 74, 6218

Burbine, T. H., Meibom, A., Binzel, R. P. 1996, Meteoritics, 31, 607

Binzel, R. P., Masi, G., \& Foglia, S. 2006, BAAS, 627, 38

Bottke, W. F., Nesvornỳ, D., Grimm, R. E., et al. 2006, Nature, 439, 821

Buckley, D. A. H., Burgh, E. B., Cottrell, P. L., et al. 2006, SPIE Conf. Ser., 6269

Burgh, E. B., Nordsieck, K. H., Kobulnicky, H. A., et al. 2003, Instrument Design and Performance for Optical/Infrared Ground-based Telescopes, Proc. SPIE, 4846, 1463

Carporzen, L., Weiss, B. P., Elkins-Tanton, L. T., et al. 2011, Proc. National Academy of Sciences, 108, 6386

Carvano, J. M., Hasselmann, P. H., Lazzaro, D., et al. 2010, A\&A, 510, A43

Chapman, C. R. 2004, Ann. Rev. Earth Planet. Sci. 32, 539

Crawford, S. M., Still, M., Schellart, P., et al. 2010, SPIE Conf. Ser., 7737, 25

DeMeo, F. E., Binzel, R. P., Slivan, S. M., et al. 2009, Icarus, 202, 160

Dufour, P., Kilic, M., Fontaine, G., et al. 2010, ApJ, 719, 803

Goldstein, J. I., \& Short, J. M. 1967, Geochim. Cosmochim. Acta, 31, 1733

Hammergren, M., Gyuk, G., Puckett, A., et al. 2006

[arXiv: astro-ph/0609420]
Ivezic, Ž., Jurić, M., Lupton, R. H., et al. 2002, In Survey and Other Telescope Technologies and Discoveries, eds., T. J. Anthony, \& W. Sidney, Proc. SPIE, 4836, 98

Jura, M., Xu, S., Klein, B., et al. 2012, ApJ, 750, 69

Jurić, M., Ivezić, Ž., Lupton, R. H., et al. 2002, ApJ, 124, 1776

Kelley, M. S., \& Gaffey, M. J. 2000, Icarus, 144, 27

Kobulnicky, H. A., Nordsieck, K. H., Burgh E. B., et al. 2003, Instrument Design and Performance for Optical/Infrared Ground-based Telescopes, Proc. SPIE, 4841, 1634

Kohout, T., Gritsevich, M., Grokhovsky, VI., et al. 2014, Icarus, 228, 78

Lazzaro, D. 2009, Rev. Mex. Astron. Astrofis., 35, 1

Masiero, J. R., Mainzer, A. K., Grav, T., et al. 2011, ApJ, 741, 68

Moskovitz, N. A., Jedicke, R., Gaidos, E., et al. 2008a, Icarus, 198, 77

Moskovitz, N. A., Lawrence, S., Jedicke, R., et al. 2008b, ApJ, 682, L57

Mothé-Diniz, T., Roig, F., \& Carvano, J. M. 2005, Icarus, 174, 54

Oszkiewicz, D. A., Muinonen, K., Bowell, E., et al. 2011, J. Quant. Spectr. Rad. Transf., 112, 1919

Oszkiewicz, D. A., Bowell, E., Wasserman L. H., et al. 2012 Icarus 219, 283

Polishook, D., Moskovitz, N., Binzel, R. P., et al. 2013, Icarus, 233, 9

Popescu, M., Birlan, M., \& Nedelcu, D. A. 2012, A\&A, 544, A130

Righter, K., \& Drake, M. J. 1997, Meteoritics, 32.6, 929

Roig, F., \& Gil-Hutton, R. 2006, Icarus, 183, 411

Roig, F., Nesvorný, D., Gil-Hutton, R., et al. 2008, Icarus, 194, 125

Russell, S. J., \& Norvig, P. 2002, Artificial intelligence: a modern approach (Pearson)

Russell, C. T., Raymond, C. A., Coradini, A., et al. 2012, Science, 336, 684

Scott, E. R. D., Greenwood, R. C., Franchi, I. A., et al. 2009, Geochim. Cosmochim. Acta, 73, 5835

Solontoi, M. R., Hammergren, M., Gyuk, G., et al. 2012, Icarus, 220, 577

Weiss, B. P., Elkins-Tanton, L. T., Barucci, M. A., et al. 2012, Planet. Space Sci., 66, 137 